\title{
VLBI observations of Jupiter with the initial test station of LOFAR and the Nançay decametric array
}

\author{
A. $\mathrm{Nigl}^{1}$, P. Zarka ${ }^{2}$, J. Kuijpers ${ }^{1}$, H. Falcke ${ }^{3}$, L. Bähren ${ }^{3}$, and L. Denis ${ }^{2}$ \\ 1 Department of Astrophysics (IMAPP), Radboud University Nijmegen, 6535 ED Nijmegen, The Netherlands \\ e-mail: anigl@astro.ru.nl \\ 2 LESIA, CNRS/Observatoire de Paris, 92195 Meudon, France \\ 3 ASTRON, 7990 AA Dwingeloo, The Netherlands \\ Received 31 January 2007 / Accepted 31 May 2007
}

\section{ABSTRACT}

\begin{abstract}
Aims. To demonstrate and test the capability of the next generation of low-frequency radio telescopes to perform high resolution observations across intra-continental baselines. Jupiter's strong burst emission is used to perform broadband full signal cross-correlations on time intervals of up to hundreds of milliseconds.

Methods. Broadband VLBI observations at about $20 \mathrm{MHz}$ on a baseline of $\sim 50000$ wavelengths were performed to achieve arcsecond angular resolution. LOFAR's Initial Test Station (LOFAR/ITS, The Netherlands) and the Nançay Decametric Array (NDA, France) digitize the measured electric field with 12 bit and 14 bit in a $40 \mathrm{MHz}$ baseband. The fine structure in Jupiter's signal was used for data synchronization prior to correlation on the time-series data.

Results. Strong emission from Jupiter was detected during snapshots of a few seconds and detailed features down to microsecond time-scales were identified in dynamic spectra. Correlations of Jupiter's burst emission returned strong fringes on $1 \mathrm{~ms}$ time-scales over channels as narrow as a hundred kilohertz bandwidth.

Conclusions. Long baseline interferometry is confirmed at low frequencies, in spite of phase shifts introduced by variations in ionospheric propagation characteristics. Phase coherence was preserved over tens to hundreds of milliseconds with a baseline of $\sim 700 \mathrm{~km}$. No significant variation with time was found in the correlations and an estimate for the fringe visibility of 1 , suggested that the source was not resolved. The upper limit on the source region size of Jupiter Io-B S-bursts corresponds to an angular resolution of $\sim 3$ arcsec. Adding remote stations to the LOFAR network at baselines up to thousand kilometers will provide 10 times higher resolution down to an arcsecond.
\end{abstract}

Key words. instrumentation: high angular resolution - methods: data analysis - methods: observational - techniques: high angular resolution - planets and satellites: general - instrumentation: detectors

\section{Introduction}

A new wave of low-frequency telescopes are about to enter astronomy. LOFAR (low frequency array) is a new-generation giant digital telescope and already its prototype (LOFAR/ITS) is capable of performing broadband and phase-sensitive interferometry with high angular, time and frequency resolution. In this paper, the nature of Jupiter's burst emission is studied at low frequencies $(<40 \mathrm{MHz})$ on a baseline of $\sim 50000$ wavelengths and an angular resolution of $\sim 3$ arcsec. We want to test over which bandwidth and time-scales coherence in Jupiter burst signal is preserved or can be reconstructed, in spite of ionospheric propagation effects causing phase variation, Faraday rotation, dispersion and refraction. We want to demonstrate that high resolution observations can be performed by cross correlations of signals from remote stations being added to the LOFAR telescope at baselines up to a thousand kilometers.

Long baseline interferometry of Jupiter's decametric emission (DAM) has been performed since 1965 on baselines up to more than $7000 \mathrm{~km}$, setting an upper limit on the source size of 0.05 arcsec (Lynch et al. 1972). Research was mainly done in the sixties and seventies using narrow-band analog recording techniques and 1 bit baseband digitization (Slee \& Higgins 1965; Dulk et al. 1967; Brown et al. 1968; Dulk 1970; Stannard et al. 1970; Lynch et al. 1972; Lynch et al. 1976).
Jupiter's DAM is produced below $40 \mathrm{MHz}$, limited by the maximum electron gyrofrequency at Jupiter's surface. The lower limit for ground-based observations is set by the plasma frequency of the Earth ionosphere between $5 \mathrm{MHz}$ and $10 \mathrm{MHz}$. The emission mechanism of Jupiter's DAM, modulated by Io, is believed to be cyclotron maser emission (Wu \& Lee 1979). The emission can be very intense up to $10^{5-6} \mathrm{Jy}$ is beamed into a thin hollow cone with its axis parallel to the local magnetic field. The hollow cone has a half-angle of $60^{\circ}$ to $90^{\circ}$ relative to the local magnetic field direction and has a cone wall thickness of a few degrees (Kaiser et al. 2000). This emission can be detected on Earth when the line of sight of the observer coincides with the wall of the hollow cone. The emission arrives in noise storms called long bursts (L-bursts) and in narrow millisecond bursts called short bursts (S-bursts). Phillips et al. $(1986,1987)$ found a first indication for source growth during L-bursts from smaller than 10 arcsec up to 60 arcsec. For S-bursts a complex microstructure at typically $\sim 40$ s period and $\sim 30 \mathrm{kHz}$ bandwidth has been observed. These sub-burst wave packets have been attributed to clouds of electrons moving along the Io flux tube (IFT) (Carr \& Reyes 1999).

Dulk (1970) put an upper limit of $400 \mathrm{~km}$ to $4000 \mathrm{~km}$ at Jupiter on the size of the DAM source at $34 \mathrm{MHz}$, which corresponds to an angular resolution of $0.1^{\prime \prime}$ to $1^{\prime \prime}$. S-burst analysis provides an upper limit of about $20 \mathrm{~km}$ on the instantaneously 
emitting source (Zarka 1996), which translates to 6 milliarcseconds at the distance of $4.25 \mathrm{AU}$.

Jupiter's DAM emission is strongly elliptically polarized. The signal at frequencies near $20 \mathrm{MHz}$ is on average $49 \%$ circularly and $87 \%$ linearly polarized (Shaposhnikov et al. 1997; Queinnec \& Zarka 2001).

For the following analysis, Jupiter was observed with the two radio telescopes LOFAR/ITS (Initial Test Station of LOFAR, The Netherlands) and NDA (Nançay Decametric Array, France), which are described in Sect. 2. In Sect. 4, correlations of simultaneous data recorded with ITS and NDA are described and then discussed in Sect. 5. Correlations were performed on dynamic spectra and on the time domain data.

\section{Radio telescopes}

\subsection{ITS}

LOFAR's Initial Test Station was located at the core site of LOFAR near the village of Exloo, The Netherlands and consists of 60 inverted-V-shaped dipole antennae distributed over five spiral arms. ITS antennae work in the lowest LOFAR frequency band from $5 \mathrm{MHz}$ to $35 \mathrm{MHz}$, which matches the emission band of Jupiter-Io emission.

The final LOFAR telescope will eventually consist of $\sim 15000$ antennae, split up between a dense core of 32 stations and 45 remote stations with 192 antennae each. These stations will be spaced on exponentially expanding shells. The antennae within the station will be placed on exponential shells with a maximum diameter of 60 meters. The total bandwidth from $30 \mathrm{MHz}$ to $240 \mathrm{MHz}$ will be covered by two different antenna types: a low band antenna (LBA) for frequencies below $80 \mathrm{MHz}$, and a high band antenna (HBA) from $110 \mathrm{MHz}$ to $240 \mathrm{MHz}$. Below $30 \mathrm{MHz}$ the gain of the low band antennae is reduced, but a fixed hardware limit is at $10 \mathrm{MHz}$. The LOFAR electronics is designed to accommodate a third type of antenna, which could cover frequencies below $30 \mathrm{MHz}$ to have a broader bandwidth for Jupiter observations. With a maximum baseline of $\sim 100 \mathrm{~km}$ between stations, all these antennae will provide LOFAR with a 2 to 20 arcsec angular resolution and millijansky sensitivity, dependent on frequency. Each dipole's signal will be amplified in an LNA (low-noise amplifier), filtered in frequency and digitized (Falcke et al. 2006). More detail on LOFAR and its test stations can be found at www. lofar. org. Additionally, a formulation of a science case with long baselines by extremely remote LOFAR stations is described by Vogt (2006).

In the case of ITS, high performance analog-to-digital converters $(\mathrm{ADC} s)$ with a dynamic range of 12 bits, supply a rate of 80 million samples per second. The sample rate results in a time resolution of $12.5 \mathrm{~ns}$ and a data rate of $\sim 160 \mathrm{MB}$ per second and dipole. Four of those ADCs are mounted on two TIMboards (twin-input module) which are accommodated in each of the 16 standard PC $s$ in the data acquisition (DAQ) container. The TIM-boards can handle two digital data streams and store each stream in 1 GB of RAM. This amount of RAM limits the DAQ to 6.7 s. From 2005 on, 30 antennae measured dual polarization, since two orthogonal dipoles were mounted in each antenna structure. Each antenna detects the whole sky with a primary beam of about $90^{\circ}$ full width half maximum (FWHM) centered on the zenith. Using digital beam-forming the array can be simultaneously pointed at several sources in the sky. For ITS the digital beam-forming was performed off-line and is described in Sect. 3. The absolute timing of ITS was updated with Network Time Protocol (NTP). The beam size of ITS at the zenith results in an angular resolution of $3^{\circ}$, at a wavelength of 10 meters $(30 \mathrm{MHz})$ and for a maximum baseline of $180 \mathrm{~m}$.

\subsection{NDA}

The Nançay Decametric Array (www.obs-nancay. fr/dam) is a phased array made up of 144 antennae. Each antenna consists of eight conducting wires (monopoles) wound up on vertical support cables on a conical surface. Each opposite pair of monopoles is connected as a spiral dipole. The array is split in two groups of 72 left-handed and 72 right-handed wound antennae to measure circularly polarized radio waves. All 72 antennae of one cluster measure the same polarization direction and can be phased up with an analog beam-former. A full description of the array can be found in Boischot et al. (1980).

The receiver used for the observations in 2005 provided the same type of data as ITs by sampling in the first Nyquist zone with $80 \mathrm{MHz}$ over a frequency band from $8 \mathrm{MHz}$ to $40 \mathrm{MHz}$. A DAQ board (Signatec PDA14) was used for the digitization with a 14-bit ADC inserted in a powerful PC, which allows continuous DAQ of a stream of 80 million samples per second. These data are written as short integers (16-bit) to disc. The receiver allows full signal detection below $40 \mathrm{MHz}$ (a low-pass filter at $40 \mathrm{MHz}$ and high-pass filter at $8 \mathrm{MHz}, 16 \mathrm{MHz}$ or $24 \mathrm{MHz}$ are used) without use of a local oscillator for frequency down conversion. Absolute timing of the instrument is provided by a receiver of the Global Positioning System (GPS).

\section{Observations}

Triggered by NDA, we took simultaneous datasets during periods of Jupiter burst emission. Simultaneous Jupiter snapshots of $6.7 \mathrm{~s}(1 \mathrm{~GB})$ at ITS and $20 \mathrm{~s}(\sim 3 \mathrm{~GB})$ at NDA were recorded. The trigger was manually executed at NDA while monitoring the real-time display of NDA for actual burst activity. The realtime monitor produces and displays dynamic spectra at a rate of one spectrum per second. The monitor can also be found online with a $30 \mathrm{~s}$ refreshment time (www.obs-nancay. fr/dam/ dam_visu.html). The day of observation was selected from NDA's monthly probability plots (www.obs-nancay.fr/dam/ dam_proba.htm, Leblanc et al. 1993).

Both telescopes recorded the full signal during overlapping periods. The data taken with NDA are available in digital format and contain the digitized output of the analog-formed beam in the direction of Jupiter. For ITS the raw digitized data for all antennae were recorded. Total intensity was not calculated due to uncertain relative gain calibration. Since a single dipole antenna measured the whole sky at the same time, it detected also the sky background as well as strong radio frequency interference (RFI). The resulting high noise level makes it impossible to observe Jupiter's emission in one antenna with enough contrast. The phasing of the antennae by digital beam-forming, in the direction of Jupiter, was performed to increase the signal-to-noise ratio by suppressing the RFI coming from the horizon.

To beam-form and display the data, the following steps were performed. First, a Fourier transform, however the computation time of the fast Fourier transform (FFT) increases as $N \log N$, where $N$ is the number of samples. Thus, splitting the antenna data in time-blocks is more efficient than processing one long time-series. However, the splitting requires extra bookkeeping when reconstructing the beam-formed time-series needed for displaying and for signal correlation.

Second, a Hamming window was applied to the raw data blocks to keep the leakage in frequency domain into 


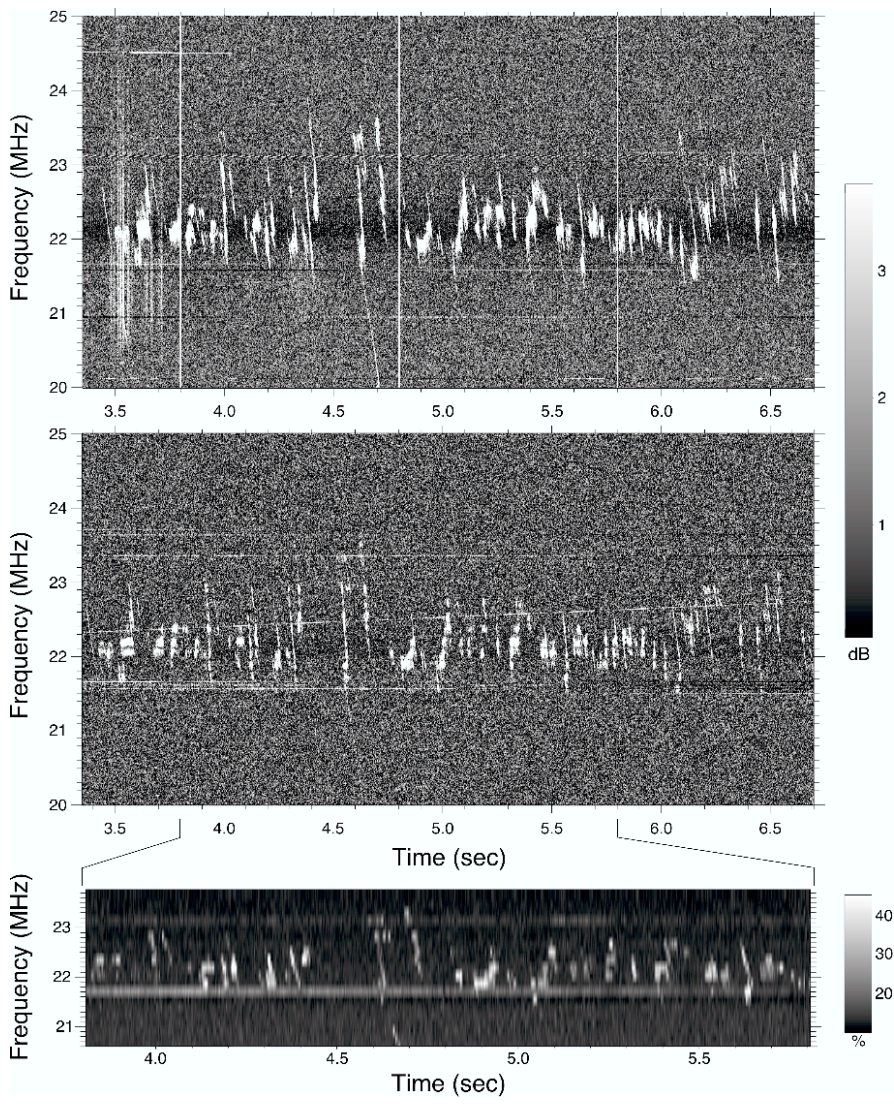

Fig. 1. Dynamic spectra of a simultaneous observation with the Nançay Decametric Array (NDA) [TOP] and LOFAR's Initial Test Station (LOFAR/ITS) [CENTER], on November 30, 2005 at 07:15:12 UTC for a duration of $\sim 3.5 \mathrm{~s}$. Jovian S-bursts show up as the discrete structures mainly confined to the band $20.7 \mathrm{MHz}$ to $23.9 \mathrm{MHz}$. Dark horizontal Faraday fringes are superimposed on emission observed at ITS. Vertical lines in the NDA spectrum are $5 \mathrm{~ms}$ pulses intentionally added every second to the data for timing purposes. Horizontal lines are caused by radio frequency interference (RFI). The third panel [BOTTOM] shows the resulting cross correlation coefficients versus time and frequency, computed from data blocks of $2.5 \mathrm{~ms}$ duration and $\sim 150 \mathrm{kHz}$ bandwidth.

neighbouring channels as low as possible. Third, the FFT was applied on blocks of 16384 samples. Fourth, the interesting band or bands were selected from the frequency domain. Fifth, the beam-forming phases were applied to each frequency channel. The beam-forming phases correct the differential light travel time to each dipole-element relative to a reference position or antenna. They are calculated for the exact fractional sample delay, that shifts the antenna data for the exact desired beam direction. The chosen block size determined a time resolution of $0.2 \mathrm{~ms}$ and a spectral resolution of $5 \mathrm{kHz}$. After averaging all the phaseshifted antenna spectra the resulting beam-spectra were stacked for each block and displayed in a dynamic spectrum. The process of true time-delay beam steering by phase-shifting and element averaging on ADC data is called digital beam-forming.

For the reconstruction of the time-series, the beam-formed blocks had to partly overlap. The start and end of each beamformed block will not contain data from all antennae, due to the delays applied to each antenna during beam-forming. For the resulting beam-formed time-series, ultimately only intervals over which delay-shifted data from all antennae exist are usable.

For visual comparison of the simultaneous data, the dynamic power spectra from the two telescopes are plotted in Fig. 1. The

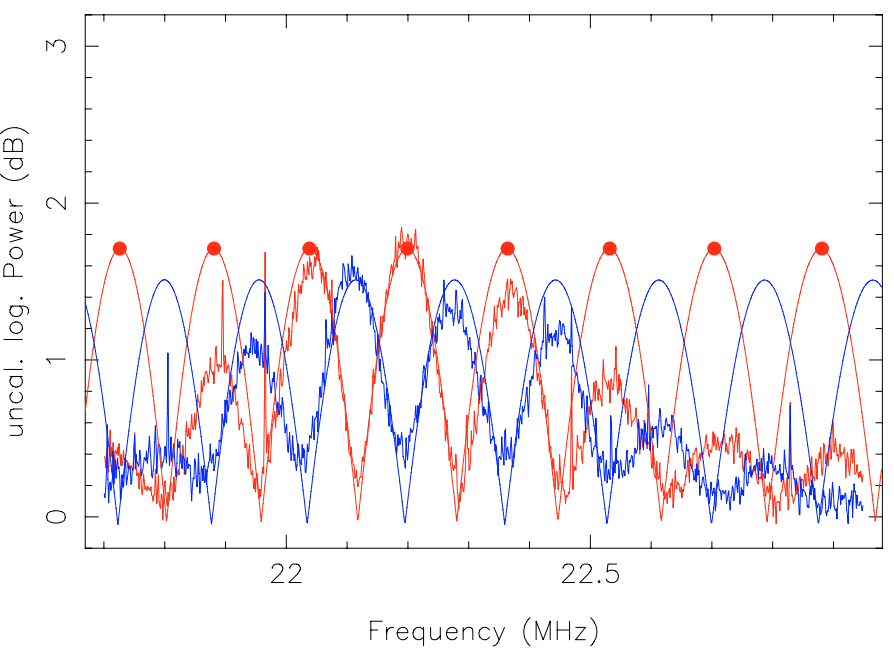

Fig. 2. Spectra of the two linear polarizations of LOFAR's Initial Test Station (LOFAR/ITS) (red: E-W antennae dipole beam and blue: N-S antennae dipole beam). The best-fit sine function (in frequency, not intensity) of the computed Faraday angle is superimposed to both ITS spectra. For the East-West polarization direction the maxima of the Faraday fringes are indicated with dots.

48 antennae of ITS and the 72 antennae of NDA were beamformed in the direction of Jupiter. The spectra were computed with $0.2 \mathrm{~ms}$ time resolution and consequently a spectral resolution of $4.9 \mathrm{kHz}$. The Jupiter signal received was expected between $20 \mathrm{MHz}$ and $30 \mathrm{MHz}$, where only a few narrow band lines contaminate the spectra. Below $20 \mathrm{MHz}$ strong RFI is dominating the spectra and above $30 \mathrm{MHz}$ the bandpass filter of ITS rolls off. The Jupiter-Io S-burst emission is clearly visible between $20.7 \mathrm{MHz}$ to $23.9 \mathrm{MHz}$ and several identical features can be identified. The emission drifts in frequency, which translates to a movement of the source region along Jupiter's magnetic field lines. These S-bursts last for a fraction of a second down to a few milliseconds. The horizontal bands in the ITS plot are due to Faraday rotation. The power spectra of the two perpendicular polarization directions of ITS show strong Faraday fringes (see Fig. 2). The spectra have a spectral resolution of $1.2 \mathrm{kHz}$ and are integrated over $6.7 \mathrm{~s}$. The plotted frequency range from $21.7 \mathrm{MHz}$ to $22.95 \mathrm{MHz}$ covers the strong Jupiter burst emission and contains only little RFI (narrow vertical lines). The Faraday fringes were fitted with a sinusoidal function according to Faraday's law. The resulting rotation measure of $R M=(1.15 \pm 0.07) \mathrm{rad} \mathrm{m}^{-2}$ is in good agreement with the rotation measure determined from total electron content values provided by the GPS. For the East-West polarization direction, the maxima of the Faraday fringes are indicated with dots. The width of the Faraday fringes was measured as $100 \mathrm{kHz}$ to $150 \mathrm{kHz}$. Around the frequencies of the eight maxima, the signal was filtered for the cross correlations described in Sect. 4.3. For a more detailed analysis of the Faraday rotation and previous results see Nigl et al. (2005).

\section{Long baseline interferometry}

For this work, Jupiter was observed with ITS and NDA on a baseline $D$ of $702 \mathrm{~km}$, corresponding to an angular resolution $\theta$ of $3.3^{\prime \prime}$ at a frequency of $v=30 \mathrm{MHz}$. For this resolution, Jupiter burst emission regions can be considered as point sources. The smallest structure that could be resolved on Jupiter at the time of 


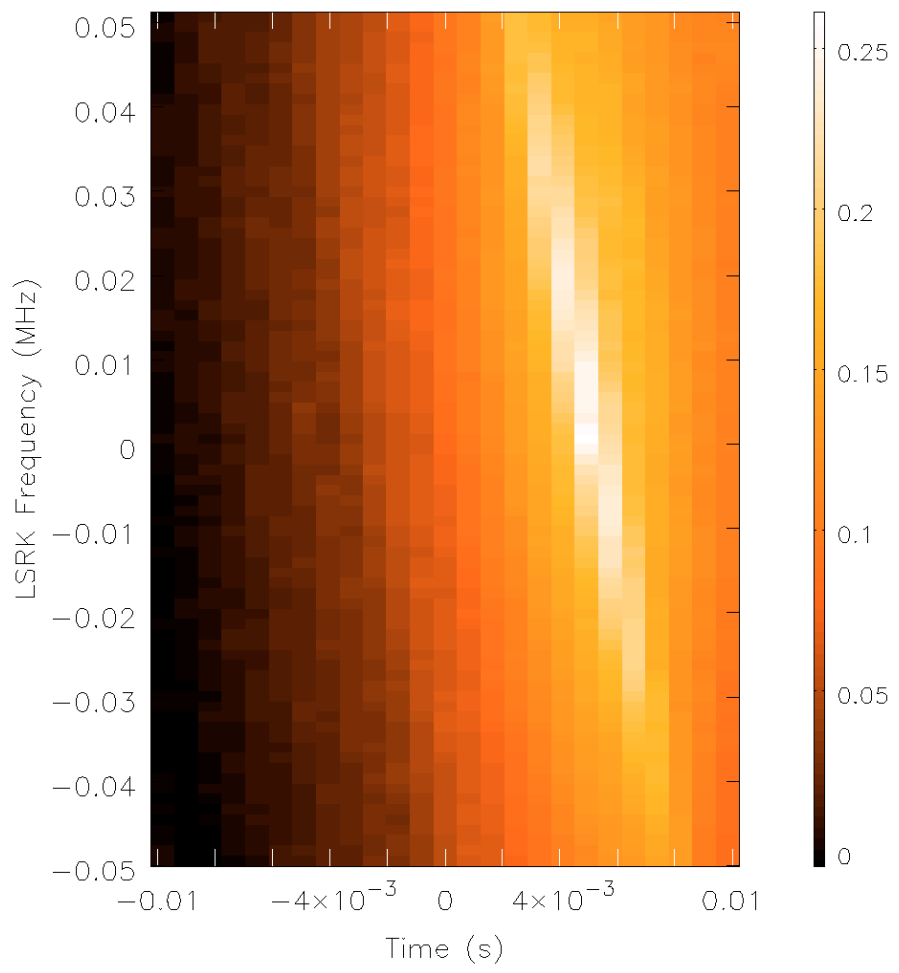

Fig. 3. Correlation coefficient matrix for two dynamic spectra slices. The intensity in the image displays the cross correlation coefficient labeled with the color wedge on the right side of the plot.

the observation was $13600 \mathrm{~km}$ in diameter, which is four times the size of Io.

\subsection{D Cross correlation of dynamic spectra}

The synchronization of the absolute timing of ITS and NDA data was of the order of one second. In order to restrict the size of the time window for correlation, a normalized 2D cross correlation of the dynamic spectra shown in Fig. 1 was performed. This cross correlation provided a coarse fringe search for the time delay and an offset in frequency between the two datasets, which is called a clock model in VLBI terminology. Before correlation, the dynamic spectra have to be generated with high time resolution for measuring the time delay, and with high spectral resolution for determining the frequency offset. The higher the resolution in time, the lower in frequency, maintaining a time-bandwidth product of unity $(\mathrm{d} v \times \mathrm{d} t=1)$. Thus for high time resolution, fine spectral structure is lost, which reduces the quality of the subsequent $2 \mathrm{D}$ cross correlation, but gives access to an accurate time delay. An offset in frequency can be found by choosing high spectral resolution.

For the observation performed on November 30, 2005 at 07:15:12 UTC, the result of a $2 \mathrm{D}$ cross correlation, of two slices of the dynamic spectra from ITS and NDA is given in Fig. 3. In this correlation window, maximum correlation is found at zero frequency shift and at a time delay of $4.9 \mathrm{~ms}$. The clock offset resulted in $10.0723328 \mathrm{~s}$ with a resolution of $1.6 \mu \mathrm{s}$ (ITS started to record later than NDA). This clock offset (clock model) serves as input for the time-series cross correlation. Additionally, a shift in frequency of $191 \mathrm{~Hz}$ with a resolution of $38 \mathrm{~Hz}$ was obtained, which will be explained in Sect. 4.5 as being mainly due to a difference between the sample rate of both telescopes, caused by the deviation of the ITS system from the nominal sample rate.
The sample rate was measured for NDA with $(79999998 \pm 0.5)$ samples per second differing only slightly from the nominal sample rate of 80 megasamples per second. This difference causes an offset from the expected and reconstructed frequency in the dynamic spectrum of $+0.6 \mathrm{~Hz}$ (for NDA at $22.3 \mathrm{MHz}$ ). Furthermore, due to Earth rotation, the radial speed of the two telescopes with respect to Jupiter at the time of the observation differed by $38 \mathrm{~m} \mathrm{~s}^{-1}$, with ITS moving towards Jupiter as seen from NDA. Therefore the ITS signal was stronger Doppler shifted to higher frequencies by $2.8 \mathrm{~Hz}$. Both frequency offsets lie within the frequency offset resulting from the $2 \mathrm{D}$ cross correlation above.

\subsection{Coherence time and coherence bandwidth}

Since emission from Jupiter arrives in bursts, the best signal-tonose ratio is achieved by correlating with integration time and frequency resolution matched to the duration and bandwidth of the bursts. The emitting electron bunches at Jupiter are traveling along the IFT at several thousands to several tens of thousands of kilometers per second. The emission is received as subpulses (wave packets) with about $40 \mu$ s to $90 \mu$ s duration and a minimum bandwidth of $30 \mathrm{kHz}$, as it has been observed by Carr $\&$ Reyes (1999). Therefore the blocks for correlation should be much longer than a wave packet of $90 \mu \mathrm{s}$ (thus $\simeq 1 \mathrm{~ms}$ ), and have a frequency resolution coarser than $30 \mathrm{kHz}$, but not more than the Faraday fringe spacing of $150 \mathrm{kHz}$. The upper limit for the correlation time window is dependent on the time-scales of changes in the Earth ionosphere and has yet to be identified. The discussed limits constrain the optimum window for the cross correlation.

\subsection{Cross correlation of time series}

The final cross correlation was done in the frequency domain following Parseval's Theorem. The fringe amplitude was calculated with the so-called fringe correlation method as the square root of the sum of the squares of the quadrature correlations (compare Lynch et al. 1976). The cosine component was obtained by a second cross correlation with a quarter-period shift at the center frequency of the selected subband introduced to one of the time series. The quarter-period shift was applied in the frequency domain as a phase-gradient, which enables shifts by a fraction of a time sample.

We used the cross correlation theorem, which performs the cross correlation of one block, for all possible lags, by only three Fourier transformations and one multiplication of the two data arrays. The lags in the cross correlation result in sample steps of $12.5 \mathrm{~ns}$.

Since the observed Jupiter-Io emission is not continuous, noise gating was applied and only time blocks with received power exceeding the noise-floor were added after cross correlation according to the fringe correlation method. Integrating about one thousand $1 \mathrm{~ms}$ blocks containing burst emission at eight bands of $75 \mathrm{kHz}$ bandwidth, resulted in an average cross correlation peak of 210 sigma significance compared to the same number of integrated blocks containing only background noise (see Fig. 4).

In total, narrow bands with bandwidths of $(30,75$ and $150) \mathrm{kHz}$ at center frequencies of $(21.73,21.88,22.04,22.20$, 22.36, 22.53, 22.70 and 22.88) $\mathrm{MHz}$ were analyzed. These frequencies were chosen as they lie at the strong maxima of the Faraday fringes from the ITS power spectra, which are shown in Fig. 2. 


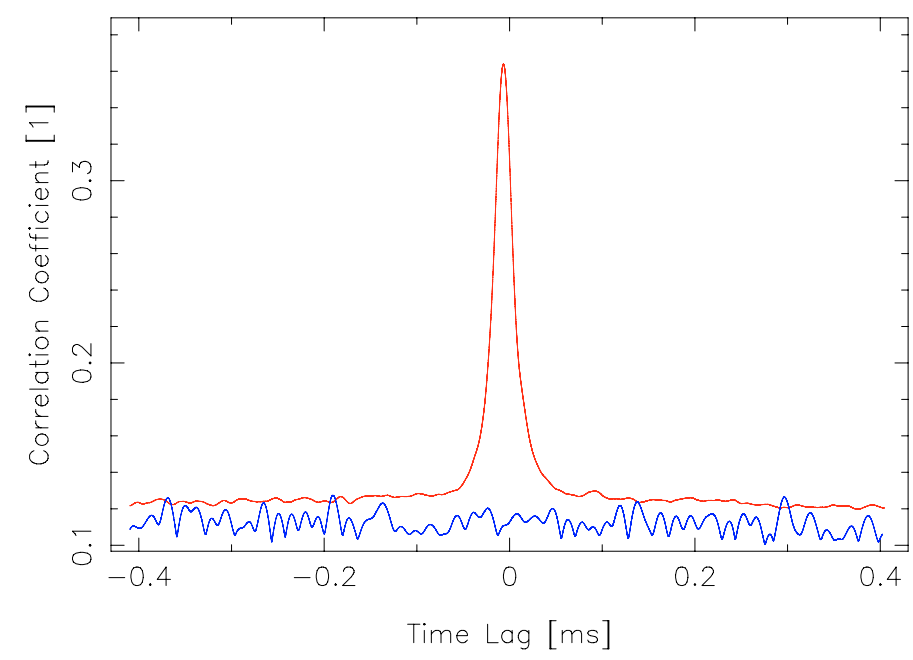

Fig. 4. Result of the time-series signal cross correlation of the NDA and ITS data sets of the Jupiter observation on 30.11.2005 at 07:15:12 UTC. The (red) peaked curve in the plot was obtained by averaging about 1000 correlated $1 \mathrm{~ms}$ time-series blocks containing Jupiter burst signal at eight bands of $75 \mathrm{kHz}$ bandwidth. The (blue) flat curve was obtained on the same number of blocks without emission. The peak maximum lies 210 sigma above the noise level with $1 \mathrm{~s}$ effective integration.

The bottom panel in Fig. 1 displays the cross-correlation coefficients in a dynamic spectrum for $2 \mathrm{~s}$ between 20.6 and 23.8 MHz, computed from $2.5 \mathrm{~ms}$ time blocks over all bright Faraday fringes.

The distribution of cross correlation coefficients of 500 correlated $1 \mathrm{~ms}$ time blocks (selected by noise gating), was maximum for a bandwidth of $30 \mathrm{kHz}$ at a center frequency of $22.53 \mathrm{MHz}$ resulting in $C=0.6 \pm 0.1$ (one sigma statistical error stated).

\subsection{Fringe visibilities}

The cross correlation coefficients of the time blocks are proportional to the fringe visibilities, which ultimately carry information on the source size, following for example Phillips et al. (1987):

$\gamma_{\mathrm{S}}=\frac{1}{G_{\tau}} k C \eta \sqrt{\frac{\left(P_{\mathrm{S} 1}+P_{\mathrm{B} 1}\right)\left(P_{\mathrm{S} 2}+P_{\mathrm{B} 2}\right)}{P_{\mathrm{S} 1} P_{\mathrm{S} 2}}}$.

Here $\gamma_{\mathrm{S}}$ is the instrument-corrected fringe visibility due to the source structure; $G_{\tau}$ is the fringe washing factor at the residual geometrical delay $\tau ; C$ is the cross correlation coefficient; $k$ and $\eta$ are instrumental decorrelation coefficients; $P_{\mathrm{S}}$ is the power from the source and $P_{\mathrm{B}}$ the power from the Galactic background both recorded at the two telescopes. The instrumental decorrelation coefficient $\eta$ accounts for any phase behavior of filters, amplifiers, cables and connectors of both telescopes together. For ITS/NDA this factor was assumed to be maximum $15 \%$ and thus $\eta=1.15$.

Since only circular polarized parts of the Jupiter signal were measured by NDA and correlated with only linear polarized components of ITS, a maximum cross correlation coefficient of $C_{\max }=\sqrt{2} / 2$ can be achieved. Therefore the second instrumental coefficient was adopted to $k=\sqrt{2}$.

The fringe washing function, $G_{\tau}$, is the Fourier transform of the receiver passband (Thompson et al. 1986). For a rectangular passband, $G_{\tau}$ is a sinc function: $G_{\tau}(\tau)=\operatorname{sinc}(\pi \Delta v \tau)$. Here, $\Delta v$ is the bandwidth of the correlated signal and $\tau$ is a residual geometrical delay, which is the difference between the true delay and the actually applied delay for the correlation: $\tau=\tau_{g}-\tau_{i}$. In the off-line correlations performed for this paper, the lag of the maximum correlation was determined for each block, which resulted in $\tau=0$ and $G=1$. The two terms (signal + noise $) /$ signal under the square root in Eq. (1) are estimated to be $\sim 1.04$ for NDA and $\sim 1.06$ for ITS. A global background correction factor of 1.1 is obtained. The resulting visibilities $\gamma_{S}$ are consistent with one, and thus with unresolved S-burst sources, as expected.

\subsection{Cross correlation lags}

The lag in which the correlation maxima laid, and hence the signal arrival time difference between the two datasets, changed by $(-8.8 \pm 0.1) \mu \mathrm{s} \mathrm{s}^{-1}$ (one sigma statistical error stated). The minus sign means that the signal arrived progressively earlier at ITS. This drift in the lag is mainly caused by a constant relative drift of the clock quartz of ITS with an accuracy of $\Delta F / F=10^{-4}$. Here $F$ is the nominal frequency and $\Delta F$ the maximum deviation including errors due to calibration, temperature variation, shock, vibration and aging over 10 years. The accuracy of the NDA clock is much better and was measured by GPS with $\Delta F / F=1.25 \times 10^{-8}$ and thus accounts for a maximum drift rate of only $12.5 \mathrm{~ns} \mathrm{~s}^{-1}$. The motion of the source with an average parallel electron velocity of $\sim 22000 \mathrm{~km} \mathrm{~s}^{-1}$ (Zarka 1996) translates to a drift of only $-367.82 \mathrm{~ns} \mathrm{~s}^{-1}$ at the Earth. Interplanetary multipath scintillation can cause delays of the order of only a few nanoseconds assuming a thin scattering sheet (Phillips et al. 1987). The Earth rotation during the short observation only accounts for $+125.17 \mathrm{~ns} \mathrm{~s}^{-1}$ and ionospheric effects change on time-scales longer than the duration of our observation and therefore were approximated by a differential change in the local slant TEC values, resulting in a contribution of $-0.032 \mathrm{~ns} \mathrm{~s}^{-1}$.

Additionally, the drift of the ITS clock causes a deviation between the sample rates of NDA and ITS of $704 \mathrm{~Hz}$. The bigger the time-window for the cross correlation, the larger the deviation in time per correlated sample gets, which reduces the quality of the correlation. Furthermore, this difference in the sample rates translates into an offset in frequency of $196 \mathrm{~Hz}$ between the dynamic spectra of both telescopes, which is consistent with the frequency offset of $191 \mathrm{~Hz}$ obtained from the 2D cross correlation of the dynamic spectra with a resolution of $38 \mathrm{~Hz}$ (Sect. 4.1).

\section{Conclusions and outlook}

Using the prototype ITS of the new generation radio telescope LOFAR and the Nançay Decametric Array, strong Io-induced Jupiter radio emission, including S-bursts, was detected over $6.7 \mathrm{~s}$ with only dozens of antennae. The beamed dynamic spectra obtained with ITS and NDA show excellent agreement (see Fig. 1). High time and frequency resolution allowed us to determine offsets by two-dimensional cross correlation of the dynamic spectra with a precision of microsecond accuracy in time and hertz accuracy in frequency. The latter method revealed a shift in frequency, due to the relative radial speed and an offset in sample frequency between the two telescopes.

The baseline of the interferometer of $702 \mathrm{~km}$ provided an angular resolution of three arcseconds. Since the emitting source region is believed to be smaller than 0.02 arcsec (Zarka 1996), the source region of the received emission remains unresolved, as confirmed by the obtained visibilities being close to one. The identified delays at the maxima of the time-series cross correlations showed a linear drift, caused by a large clock rate at ITS. Correcting for this constant drift, no significant variation was found within the observation of $6.7 \mathrm{~s}$. One second effective integration in time and several hundred kilohertz bands in frequency resulted in an 210 sigma detection above the noise level. 
This is the first time that VLBI-type observations have been performed over a broad relative bandwidth with baseband digitization, and direct correlation of signals with a large dynamic range. Cross correlation fringes on the beam-formed time-series were found on time-scales of milliseconds. As demonstrated with the ITS prototype, LOFAR will probably reach arcsecond angular resolution. LOFAR will be pioneering in being capable of performing VLBI-type observations as described in this article with millijansky sensitivity. The present results support the European extension of LOFAR with very remote stations up to several hundred kilometers. A baseline of a thousand kilometers will serve LOFAR with a spatial resolution of an arcsecond at $30 \mathrm{MHz}$. Such a resolution will enable detailed studies of the Jupiter magnetosphere as described in Zarka (2004) among many other astrophysical applications (Vogt 2006).

A follow up of these long-baseline interferometry studies is planned with NDA and LOFAR/Cs1 (Core Station 1). The follow up experiments will be performed to quantify at what duty cycle time-phase-coherence is preserved, as a function of time and frequency. Furthermore, weaker radio sources may be used in addition to Jupiter.

Acknowledgements. We wish to thank for their contribution to the observations and results presented here, the team at ASTRON for operating LOFAR/ITS, no matter what day, time or obstacle and Sebastien Hess at the Observatoire de Paris, who helped us to perform the observations with NDA.

\section{References}

Boischot, A., Rosolen, C., Aubier, M. G., et al. 1980, Icarus, 43, 399 Brown, G. W., Carr, T. D., \& Block, W. F. 1968, ApJ, 1, 89

Carr, T. D., \& Reyes, F. 1999, J. Geophys. Res., 104, 25127

Dulk, G., Rayhrer, B., \& Lawrence, R. 1967, ApJ, 150, L117

Dulk, G. A. 1970, ApJ, 159, 671

Falcke, H., van Haarlem, M. P., de Bruyn, A. G., et al. 2006, in Highlights of Astronomy, XXVIth IAU General Assembly, ed. K. A. van der Hucht, 14

Kaiser, M. L., Zarka, P., Kurth, W. S., Hospodarsky, G. B., \& Gurnett, D. A. 2000, J. Geophys. Res., 105, 16053

Leblanc, Y., Gerbault, A., Denis, L., \& Lechacheux, A. 1993, A\&A, 98, 529

Lynch, M. A., Carr, T. D., May, J., et al. 1972, ApJ, 10, 153

Lynch, M. A., Carr, T. D., \& May, J. 1976, ApJ, 207, 325

Nigl, A., Kuijpers, J., Falcke, H., Zarka, P., \& Bähren, L. 2005, in Proc. Planetary Radio Emissions VI (PREVI) ed. H. O. Rucker, S. J. Bauer, \& A. Lecacheux, 517

Phillips, J. A., Carr, T. D., Levy, J., \& Greenman, W. B. 1987, 18 MHz interferometry of Non-Io-C L-bursts

Queinnec, J., \& Zarka, P. 2001, Planet. Space Sci., 49, 365

Shaposhnikov, V. E., Kocharovsky, V. V., Kocharovsky, V. V., et al. 1997, A\&A, 326,386

Slee, O. B., \& Higgins, C. S. 1965, Radio Science, 69D, 1536

Stannard, K. M., Dulk, G. A., \& Rayhrer, B. 1970, Radio Science, 5, 1271

Thompson, A. R., Moran, J. M., \& Swenson, G. W. 1986, Interferometry and Synthesis in Radio Astronomy (A Wiley-Interscience publication)

Vogt, C. 2006, A Science Case for an extended LOFAR

Wu, C. S., \& Lee, L. C. 1979, ApJ, 230, 621

Zarka, P. 1996, Geophys. Res. Lett., 23, 125

Zarka, P. 2004, Planet. Space Sci., 52, 1455 